\title{
The Effectiveness of Cognitive-Behavioral Play Therapy (CBT) and Resiliency Based Play Therapy on Sleep Disorder in Children with Functional Abdominal Pain
}

\author{
Shekoufeh Nikneshan ${ }^{1}$ (D), Mohsen Golparvar 2,* (D), Ahmad Abedi ${ }^{3}$ (D), Peiman Nasri ${ }^{4}$ (D), \\ Fatemeh Famouri ${ }^{5}$ (iD) \\ ${ }^{1}$ Ph.D. Student, Department of Psychology, Isfahan (Khorasgan) Branch, Islamic Azad University, Isfahan, Iran \\ ${ }^{2}$ Associate Professor, Department of Psychology, Isfahan (Khorasgan) Branch, Islamic Azad University, Isfahan, Iran \\ ${ }^{3}$ Associate Professor, Department of Children with Special Needs, University of Esfahan, Isfahan, Iran \\ ${ }^{4}$ Assistant Professor, Pediatrics Department, Isfahan University of Medical Sciences, Isfahan, Iran \\ ${ }^{5}$ Associate Professor, Pediatrics Department, Isfahan University of Medical Sciences, Isfahan, Iran \\ * Corresponding author: Mohsen Golparvar, Associate Professor, Department of Psychology, Isfahan (Khorasgan) Branch, Islamic \\ Azad University, Isfahan, Iran. E-mail: drmgolparvar@gmail.com \\ How to Cite this Article: \\ Nikneshan Sh, Golparvar M, Abedi A, Nasri P, Famouri F. The Effectiveness of Cognitive-Behavioral Play Therapy (CBT) and \\ Resiliency Based Play Therapy on Sleep Disorder in Children with Functional Abdominal Pain. Iran J Rehabil Res Nurs. \\ 2020;7(1):75-85. \\ DOI: 10.29252 /ijrn.7.1.75
}

Received: 19 Dec 2019

Accepted: 09 Mar 2020

Keywords:

Resiliency Based Play Therapy

Cognitive-Behavioral Play

Therapy

Functional Abdominal Pain

Sleep Disorder

(C) 2020 Iranian Journal of

Rehabilitation Research in Nursing

\begin{abstract}
Introduction: Children with functional abdominal pain often suffer from Abdominal pain before falling asleep. This study aimed to determine the effects of resiliency based play therapy and cognitive-behavioral play therapy on sleep disorder in children with functional abdominal pain.

Methods: The research method was quasi-experimental, with pre-test, post-test, and follow-up with a control group. The research participants consisted of 24 children with functional abdominal pain aged 5-10 years old with their mothers referred to Amin Hospital Specialized Clinic in Isfahan (2018), who were purposefully selected and randomly assigned to three groups (two therapeutic groups and a control group, each group with 8 children). Bruni et al. (1996) sleep disorder Scale was used to measure the dependent variable in the pre-test, post-test, and one month follow up. Two experimental groups received a treatment course on cognitive-behavioral play therapy and resiliency based play therapy in 8 sessions, and the control group did not receive any treatment. The data were analyzed using repeated-measures analysis of variance.

Results: The results revealed that only there was a significant difference between re saliency-based play therapy and cognitive-behavioral play therapy with a control group in sleep $(P<0.05)$, there was no significant difference between resiliency based play therapy and cognitive-behavioral play therapy $(P>0.05)$.

Conclusions: Resiliency based play therapy and cognitive play therapy have been effective in recovering sleep disorder of children with functional abdominal pain. Therefore, this treatment can be used to improve sleep disorders in children with functional abdominal pain in health centers.
\end{abstract}

\section{Extended Abstract}

OBJECTIVE

hildren with functional abdominal pain often suffer from abdominal pain before falling asleep, and pain has a negative effect on sleep quality. These children have symptoms of behavioral sleep disorders, nightmares, and more daily fatigue than healthy children. Play therapy is one of the most

Copyright (C) 2020 The Author(s); Published by Iranian Journal of Rehabilitation Research in Nursing. This is an open access article, distributed under the terms of the Creative Commons Attribution-NonCommercial 4.0 International License (http://creativecommons.org/licenses /by-nc/4.0/) which permits others to copy and redistribute material just in noncommercial usages, provided the original work is properly cited. 
important therapeutic approaches for children. Cognitive-behavioral play therapy contains the principles of cognitive-behavioral therapy and play and is designed to teach children coping skills. Cognitive Behavioral interventions for behavioral childhood insomnia are effective and lead to a rapid improvement in children's sleep. However, beyond cognitivebehavioral intervention for children, it is necessary to pay attention to another part of sleep effects and other psychological variables. Research has shown that sleep affects the resiliency levels of children and adolescents. So that sleep problems would lead to reduced resiliency and potentially increasing the risk of psychopathology. In support of the role of promoting resiliency based play and its relation to sleep problems, now some studies aimed at teaching resiliency skills for children have shown success in increasing resiliency and reducing psychopathology in children. According to research evidence presented on the relationship between sleep, resilience, and cognitive-behavioral skills, In the present study, it was necessary to investigate the effect of these two interventions on sleep disorders in children with abdominal pain. This study aimed to determine the effects of resiliency based play therapy and cognitive-behavioral play therapy on sleep disorder in children with functional abdominal pain.

\section{MATERIALS AND METHODS}

The research method was quasi-experimental, with pretest, post-test, and follow-up with a control group. The research participants consisted of children with functional abdominal pain aged 5-10 years old with their mothers referred to Amin Hospital Specialized Clinic in Isfahan (2018). Twenty-four children were purposefully selected and randomly divided into three groups (two therapeutic groups and a control group, each group with eight children). Bruni et al. (1996) sleep disorder Scale was used to measure pre-test, posttest, and one month follow up. Two experimental groups received a treatment course on cognitivebehavioral play therapy and resiliency based play therapy in eight sessions, and the control group received no intervention at the end of the study. The data were analyzed using repeated-measures analysis of variance.

\section{RESULTS}

Descriptive findings showed that in the sleep disorder, the cognitive-behavioral play therapy group had a mean of 45.75 in the pre-test phase, and in the post-test and follow-up phase, the mean of this variable was changed to 35 and $34 / 87$ respectively. In the resiliency based play therapy group, the mean of sleep disorder in the pre-test phase was 45.75 , and in the post-test and follow-up phase, it was changed to 34.12 and 32 , respectively. In the control group, the mean of sleep disorder in the pretest phase was 48.75 , and in the post-test and follow-up phase, it changed to 54 and 51.78 . The results of repeated measure analysis of variance indicated that there is a significant difference between one of the therapeutic groups and the control group in sleep disorder in post-test and follow-up $(\mathrm{P}<0.001)$. The results of the posthoc Bonferroni test revealed that there was no significant difference between resilience-based play therapy and cognitive-behavioral play therapy $(\mathrm{P}>$ $0.05)$. However, there was a significant difference between the two treatments with the control group $(\mathrm{P}<$ 0.05) (Table 1).

Table 1. Results of Repeated Measures Analysis of Variance for Sleep Disturbance Variables by Intra-Group and Between-Group Analysis

\begin{tabular}{lcccccc}
\hline Analysis & Sum of Square & df & Mean Square & f & sig & Partial Eta Squared \\
\hline Inter-group & & & & & \\
$\quad$ Time (three steps) & 667.69 & 2 & 338.85 & 17.69 & 0.001 & 1 \\
$\quad$ Group x Time interaction & 907.06 & 4 & 226.76 & 11.84 & 0.001 & - \\
Error & 804.58 & 42 & 19.16 & - & & \\
Between-group & & & & & \\
$\quad$ group & 2958.11 & 2 & 1479.06 & 6.14 & -008 \\
$\quad$ Error & 5056.54 & 21 & 240.79 & - & - & - \\
\hline
\end{tabular}

\section{CONCLUSION}

In CBPT sessions, Cognitive-behavioral techniques have led to cognitive development, reduced night stress, and increased coping strategies in children. Since physiological factors may lead to sleep problems, therefore, improving the abdominal pain in children has reduced the sleep disorders of children. On the other hand, resiliency training based play therapy helps maintain positive adaptation and returns to baseline after facing adverse conditions. The child's thoughts and strengths and weaknesses can be assessed by playing. The reasons for the child's insomnia can be asked in the play and then explore possible ways to solve the problem. Through the play, the child can explore past and present events and solve problems through role- play, storytelling, or mental imagery. In the present study, the main focus of resilience-based play therapy was to enhance the sense of self-efficacy and adaptation to challenging situations for children with abdominal pain. Children with abdominal pain have lost their sense of function and self-efficacy, and instead of using problem-solving, they show their problems in other ways, including insomnia. Therefore, Resiliency-based play therapy with focusing on the child's strengths and practicing required skills leads the child to be more prepared to face the maladaptation and solving problems, making it easier for the children to solve their problems and then have a better sleep.

Resiliency based play therapy and cognitive play therapy have been effective in recovering sleep 
disorder of children with functional abdominal pain. Therefore, this treatment can be used to improve sleep disorders in children with functional abdominal pain in health centers.

\section{Ethical Considerations}

This research is taken from the doctoral dissertation on psychology and has a code of ethics from the University's Scientific Research Committee of IR.IAU.KHUISF. REC.1397.012. The authors of this study have followed the Helsinki study ethics protocols. The information of the participants in this study remains completely anonymous. All of this information will be protected after the study. Research objectives are fully described to the participants (parents of the children). They also filled out the consent form of the study.

\section{Funding or Supports}

This study had no sponsors and was conducted with a personal cost.

\section{Author's Contributions}

Ms. Shekoufeh Nikneshan's responsibility was the initial writing and preparation of the paper. Dr. Golparvar did the statistical analysis of the data and was responsible for the initial idea of the study. Dr. Abedi also had the initial idea for the study. Dr. Nasri and Dr. Famouri undertook to collect the sample.

\section{Conflict of Interest}

In this article, there was no reported any conflict of interest.

\section{Applicable Remarks}

This study by examining the role of cognitive-behavioral play therapy and resilience-based play therapy for children with functional abdominal pain will help improve the sleep of children with functional abdominal pain.

\section{Acknowledgments}

The authors would like to express our sincere appreciation to all the doctors, children, and their mothers, and the staff of Amin Biomedical clinic who helped us with this research. 


\section{اثر بخشى بازى درمانى شناختى -رفتارى و بازى درمانى مبتنى بر تاب آورى بر اختلال خواب كودكان مبتلا به درد شكمى عملكر دى}

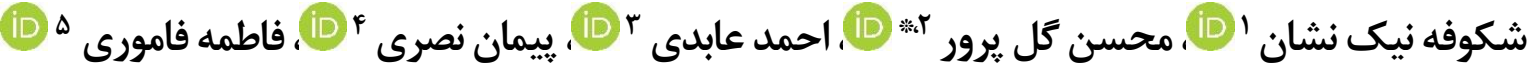

$$
\begin{aligned}
& \text { ' دانشجوى دكترى تخصصى، كروه روان شناسى، واحد اصفهان (خوراسكان)، دانشكاه آزاد اسلامى، اصفهان، ايران }
\end{aligned}
$$

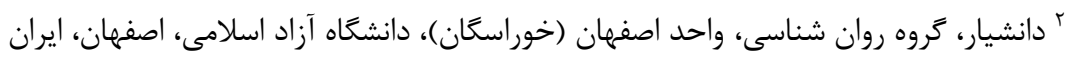

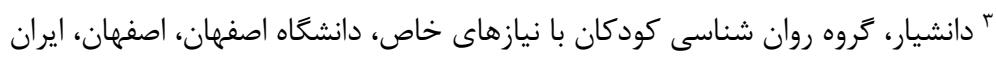

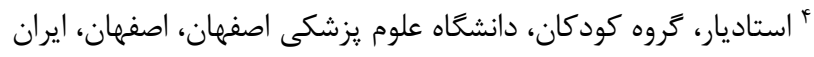

$$
\begin{aligned}
& \text { ه دانشيار، كروه كودكان، دانشكاه علوم يزشكى اصفهان، اصفهان، ايران }
\end{aligned}
$$

" نويسنده مسئول: محسن گل يرور، دانشيار، گروه روان شناسى، واحد اصفهان (خوراسگان)، دانشعاه آزاد اسلامى، اصفهان، ايران. ايميل: drmgolparvar@gmail.com

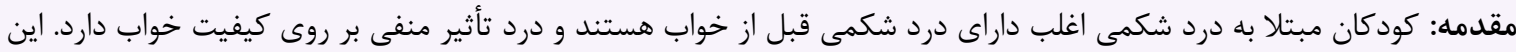

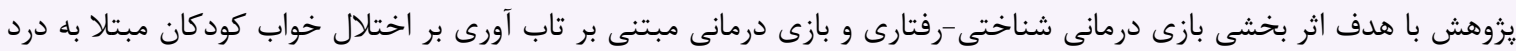
شكمى اجرا شد.

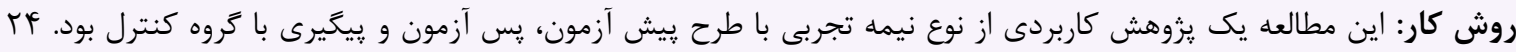

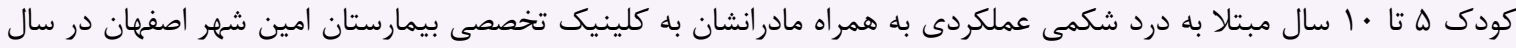

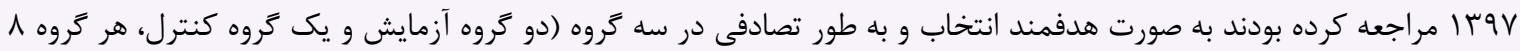

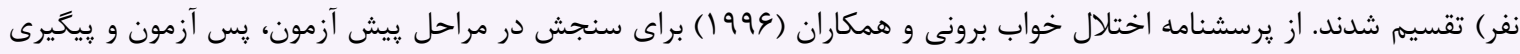

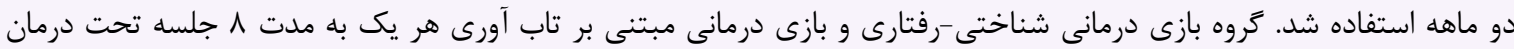

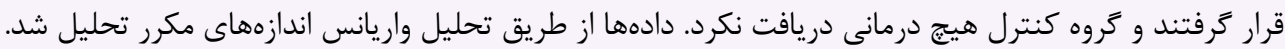

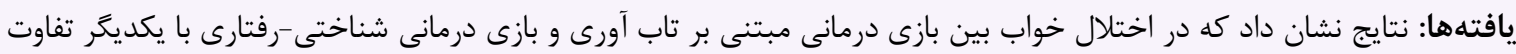

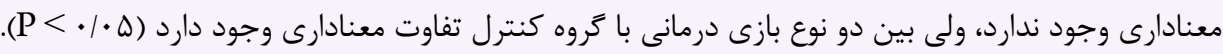

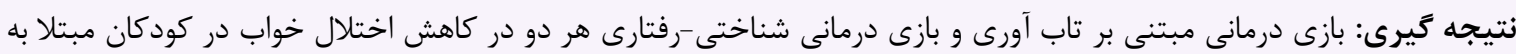

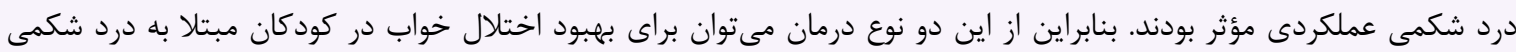

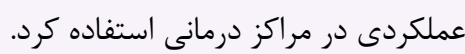
كليدوازهها: بازى درمانى مبتنى بر تاب آورىى، بازى دردمانى درمانى شناختى رفتارى، اختلال خواب، كودكان، مادران

ه/ با درصد تزارش شده، يك دوره مزمن دارد و درد مداوم مىتواند

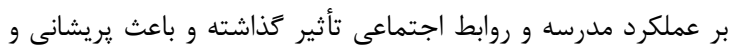

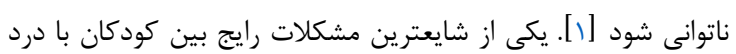

درد شكمى عملكردى يكى از بيمارىهاى دستخاه گوارش و اختلالى

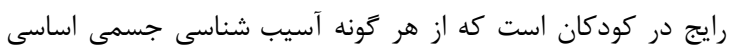

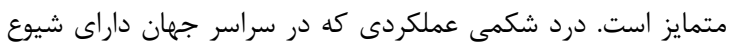


بازى درمانى در ساختارها و قالبهاى مختلف براى كودكان مبتلا به

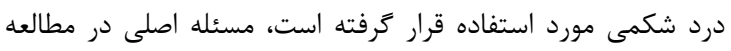

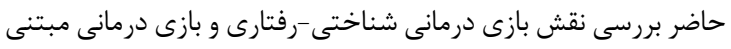

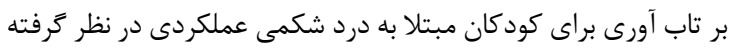

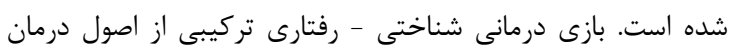

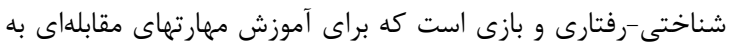

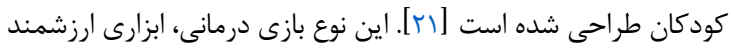

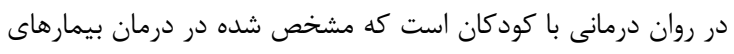

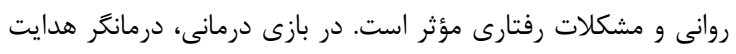

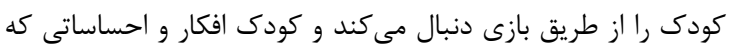

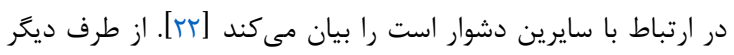

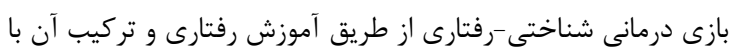

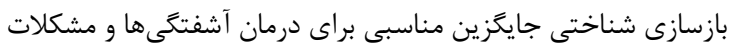

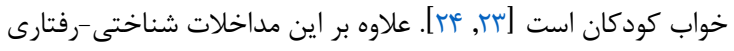

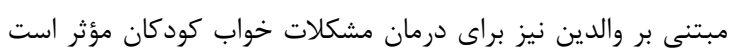

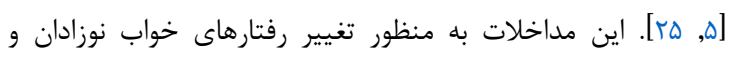

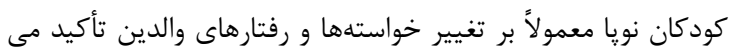

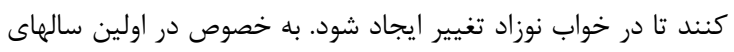

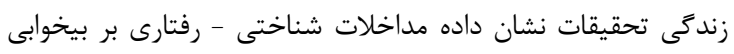

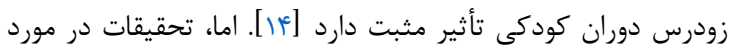

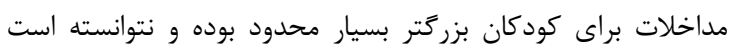

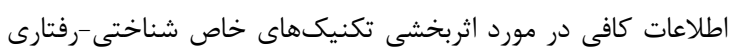

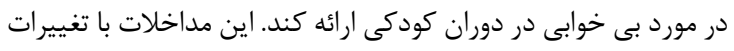

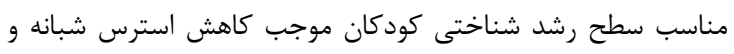

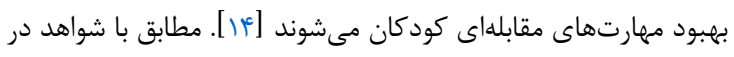
دسترس، درمان مشكلات خواب كودكان نه تنها براى خود آنها، بلكه

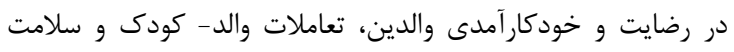

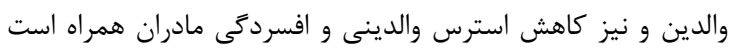

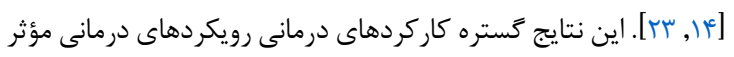

را به خوبى نشان مى دهند.

اما فراتر از مداخله شناختى -رفتارى در قالب بازئ دازى براى كودكان، لازم

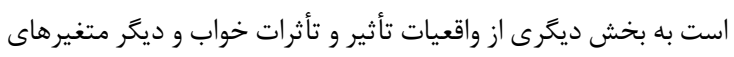

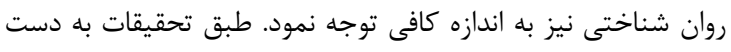

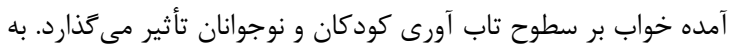
طورى كه اختلال خواب باعث كاهش تاب آورى و در نتو نتيجه افزايش

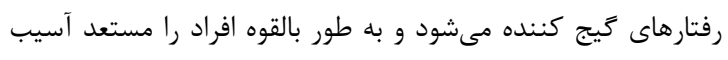

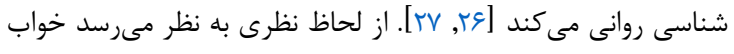

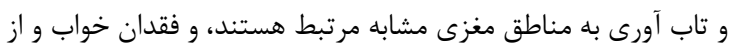

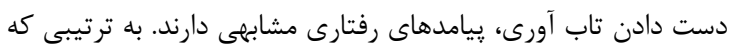

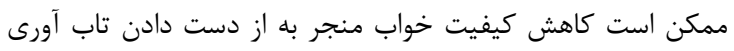

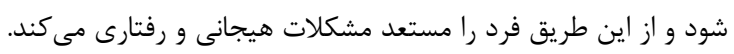

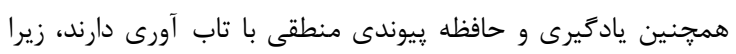

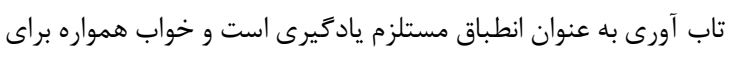

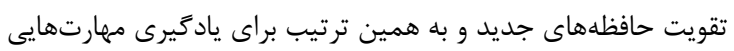

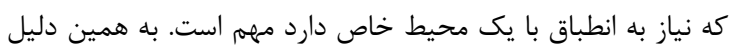

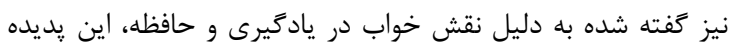

شكمى عملكردى، درد شكم قبل از شروع خواب است كه در وج درصد

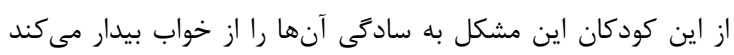

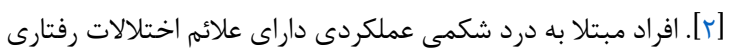

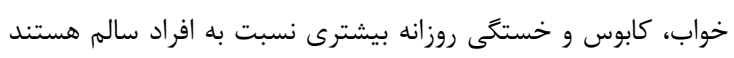

[r]

خواب نقش مهمى در عملكرد فيزيكى و روانى بهينه ايفا مى كند [ب].

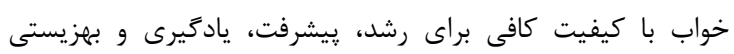

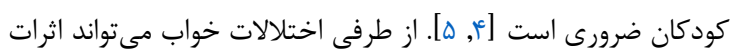

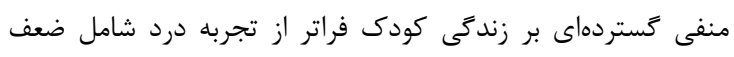

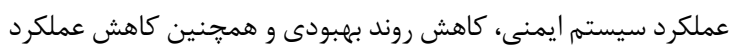

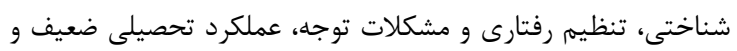
مشكلات ارتباطى كردد [ـ-1)].

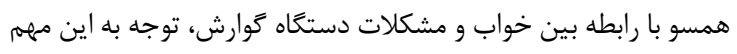

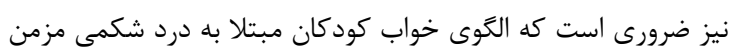

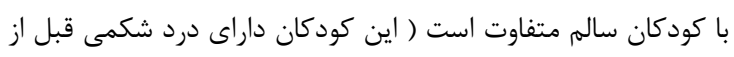

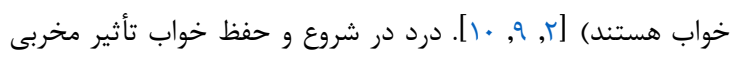

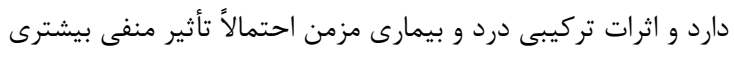

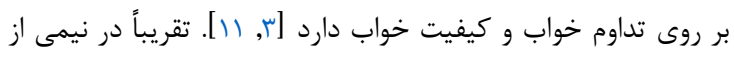

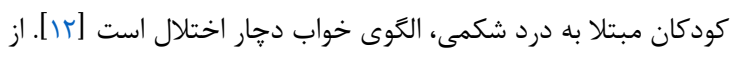

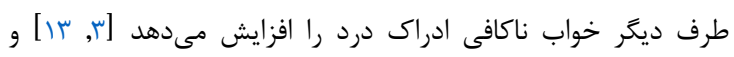

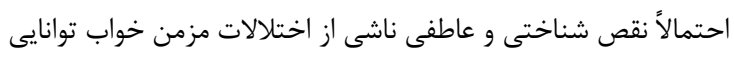

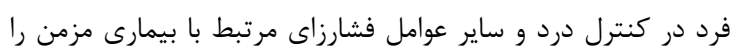

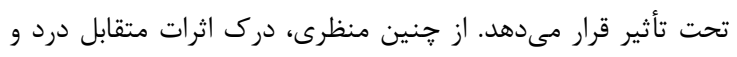

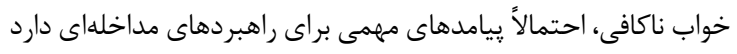

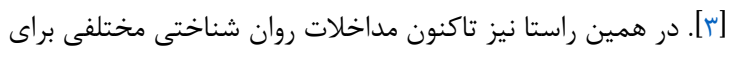

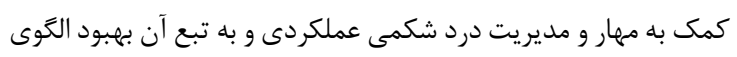

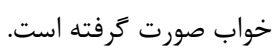

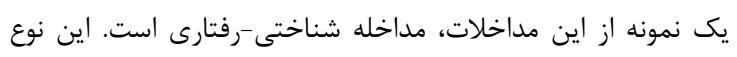

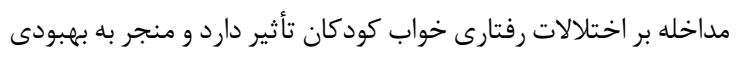

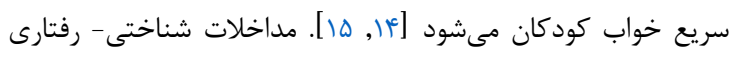
شامل رويكردهاى درمانى است كه از شيوههاى شناختى (مانند اصلاح

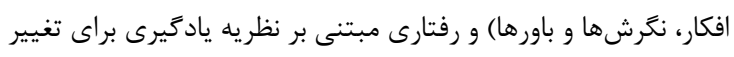

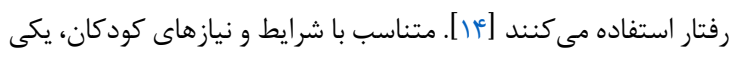

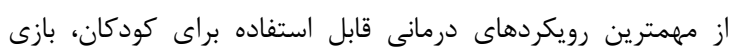

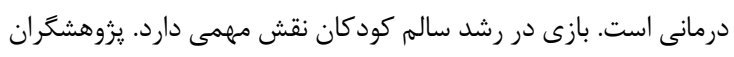

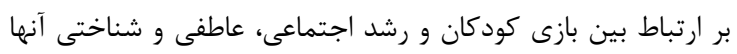

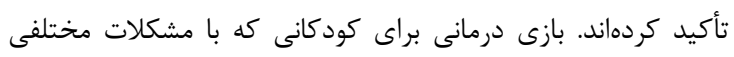

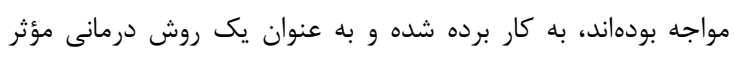

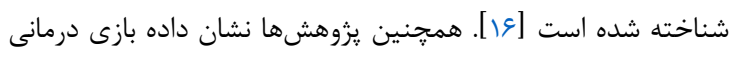

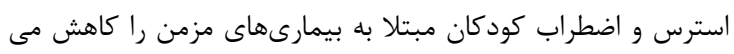

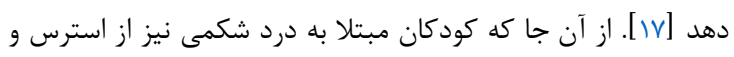

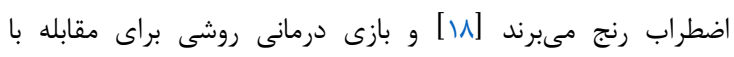

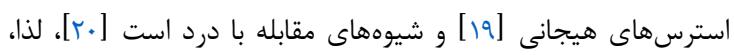

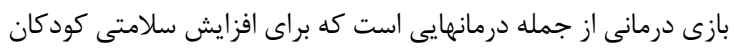

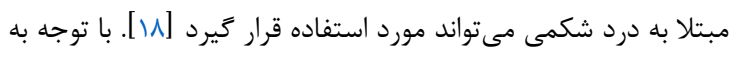
نكات مورد اشاره، با توجه به اين كه در ايران در كمتر مطالعهاى مود تاكنون 
يزووهش تحليل آمارى انجام شود و حنانجه توان آمارى ^/ • و بالاتر بود،

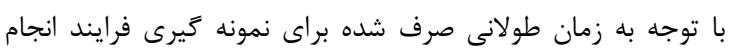

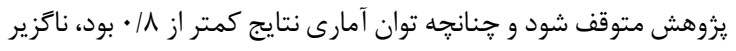

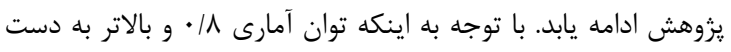

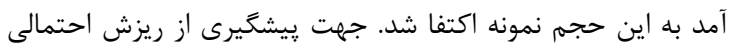

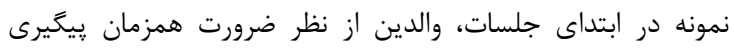

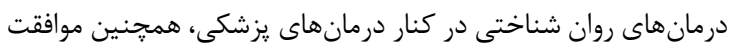

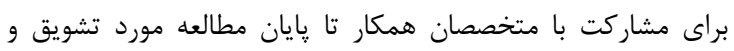

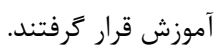

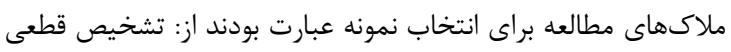

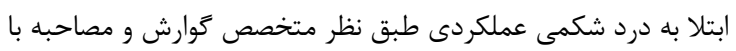

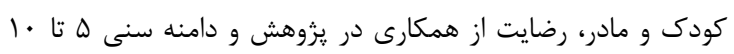

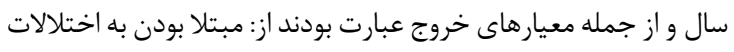

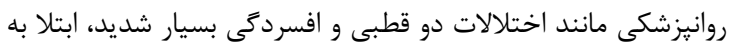

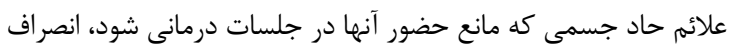

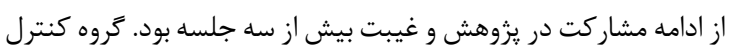

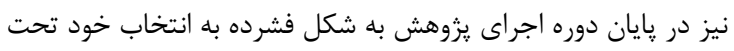

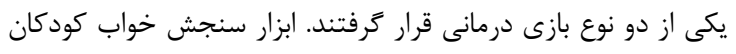

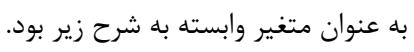

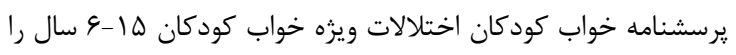

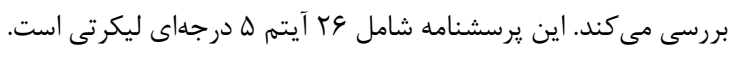

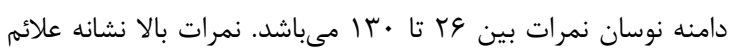

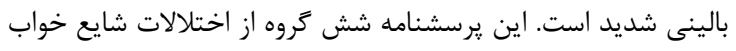

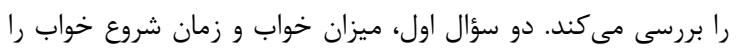

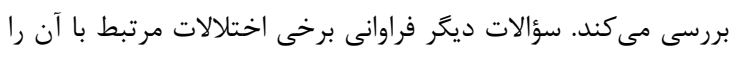

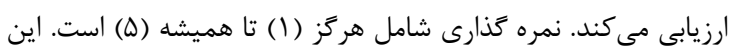

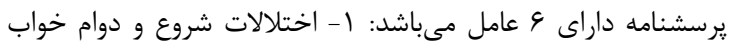

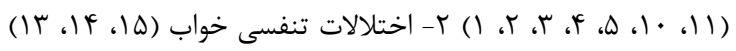

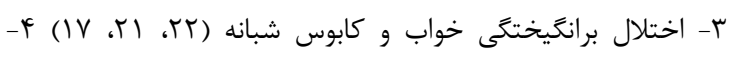

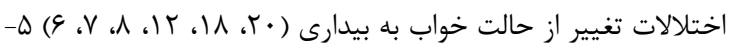

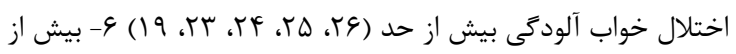

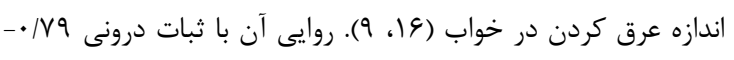

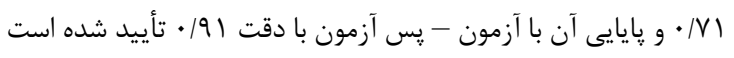

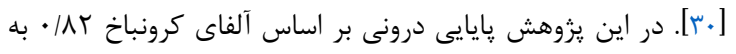

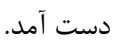

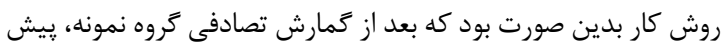

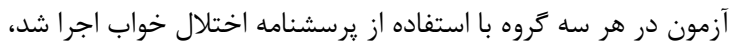

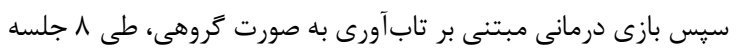

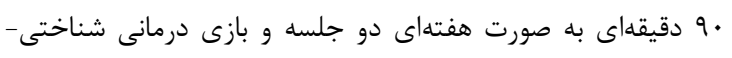

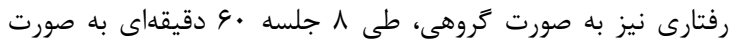

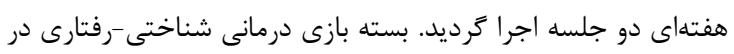

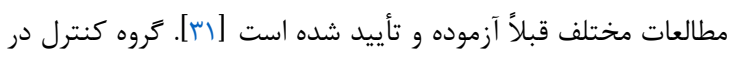

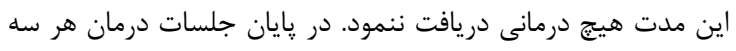

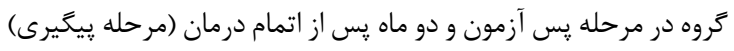

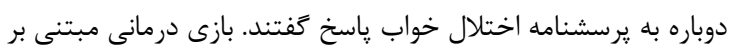

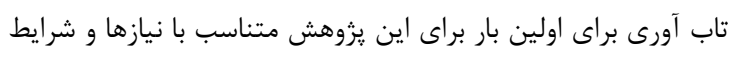

عاملى فعال در تعيين سطح تاب آورى فرد است [كҮr]. در حمايت از

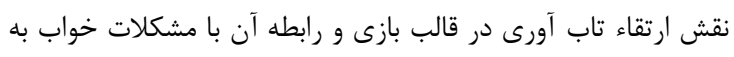

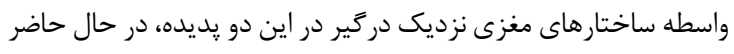

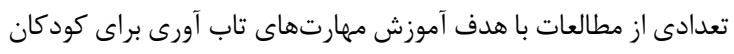

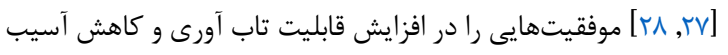

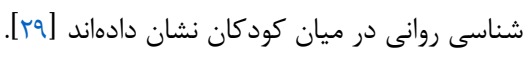

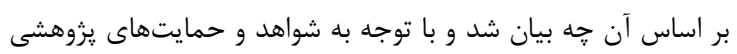

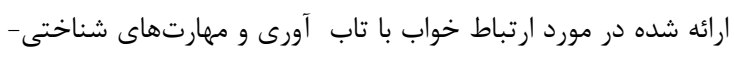

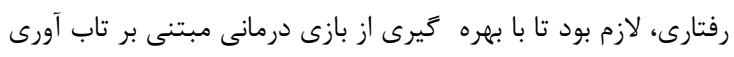

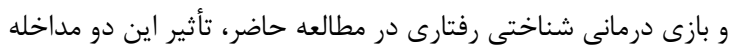

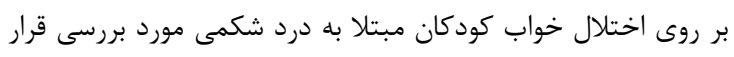

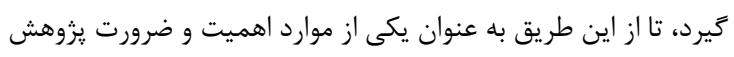

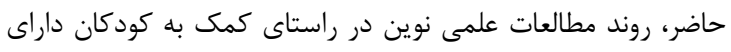

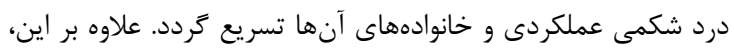

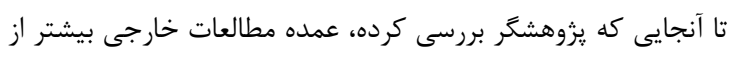

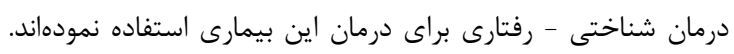

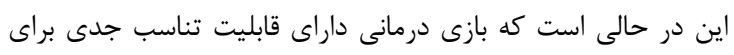

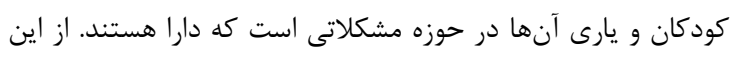

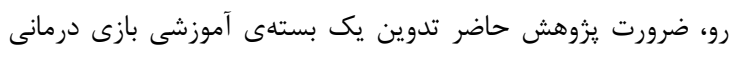

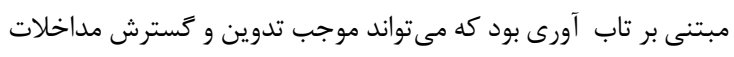

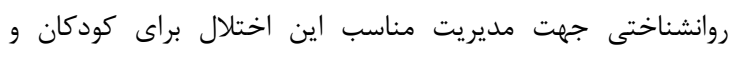

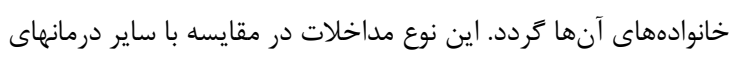

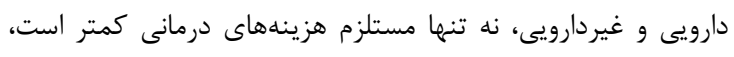

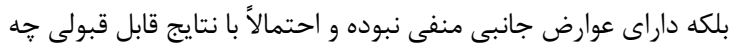

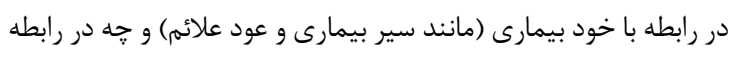

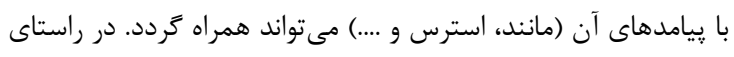

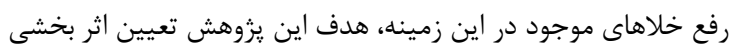

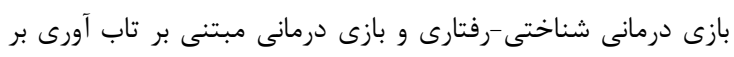
اختلال خواب كودكان مبتلا به درد شكمى عملكردى بونى بودي

\section{روش كار}

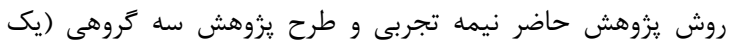

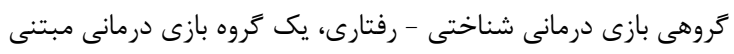

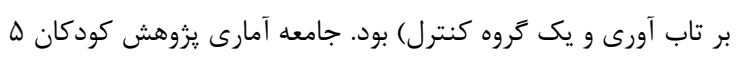

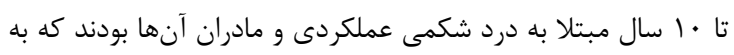

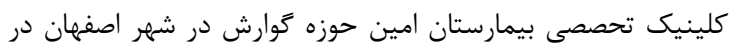

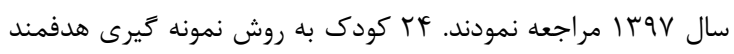

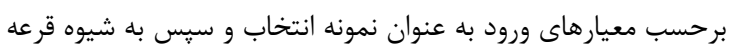

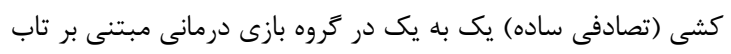

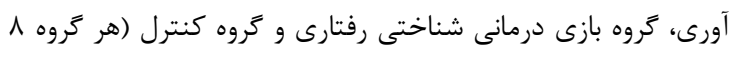

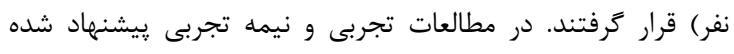

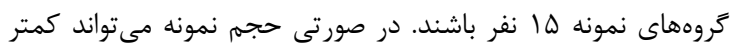

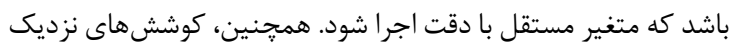

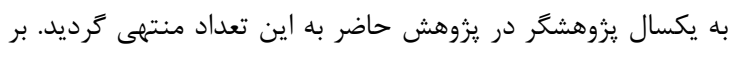

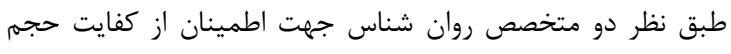
نمونه، موافقت كرديد تا بر روى تعداد \ نفر براى كرومهاى نمائ نمونه 
يروهش بود. كروه كنترل نيز در پايان دوره اجراى يُوهش به شكل

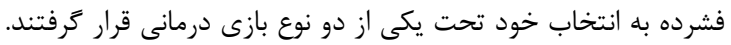

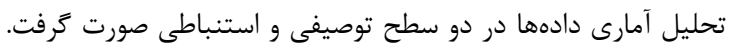

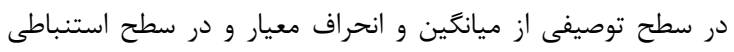

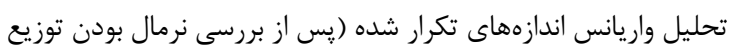

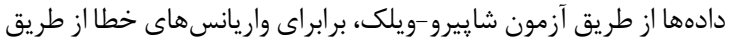

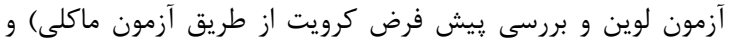

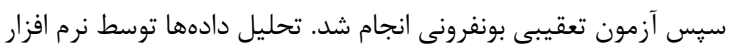
نسخه SPSS

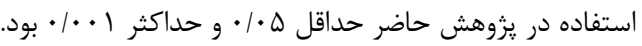

كودكان داراى درد شكمى بر مبناى متون علمى اعم از كتب و مقالات

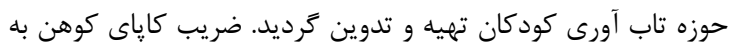

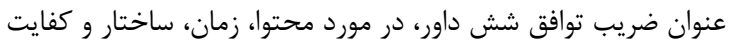

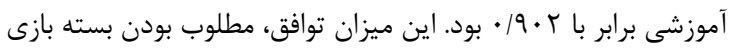

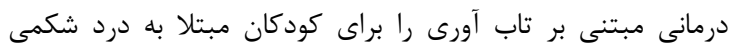

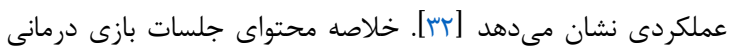

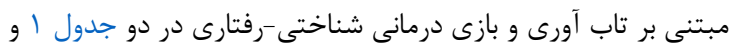
r ارائه شده است.

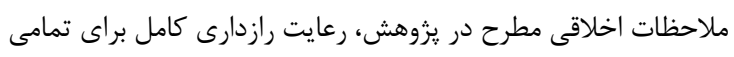

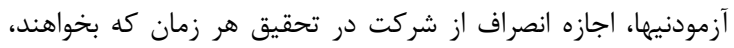

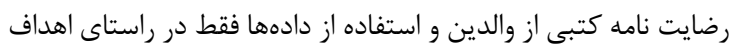

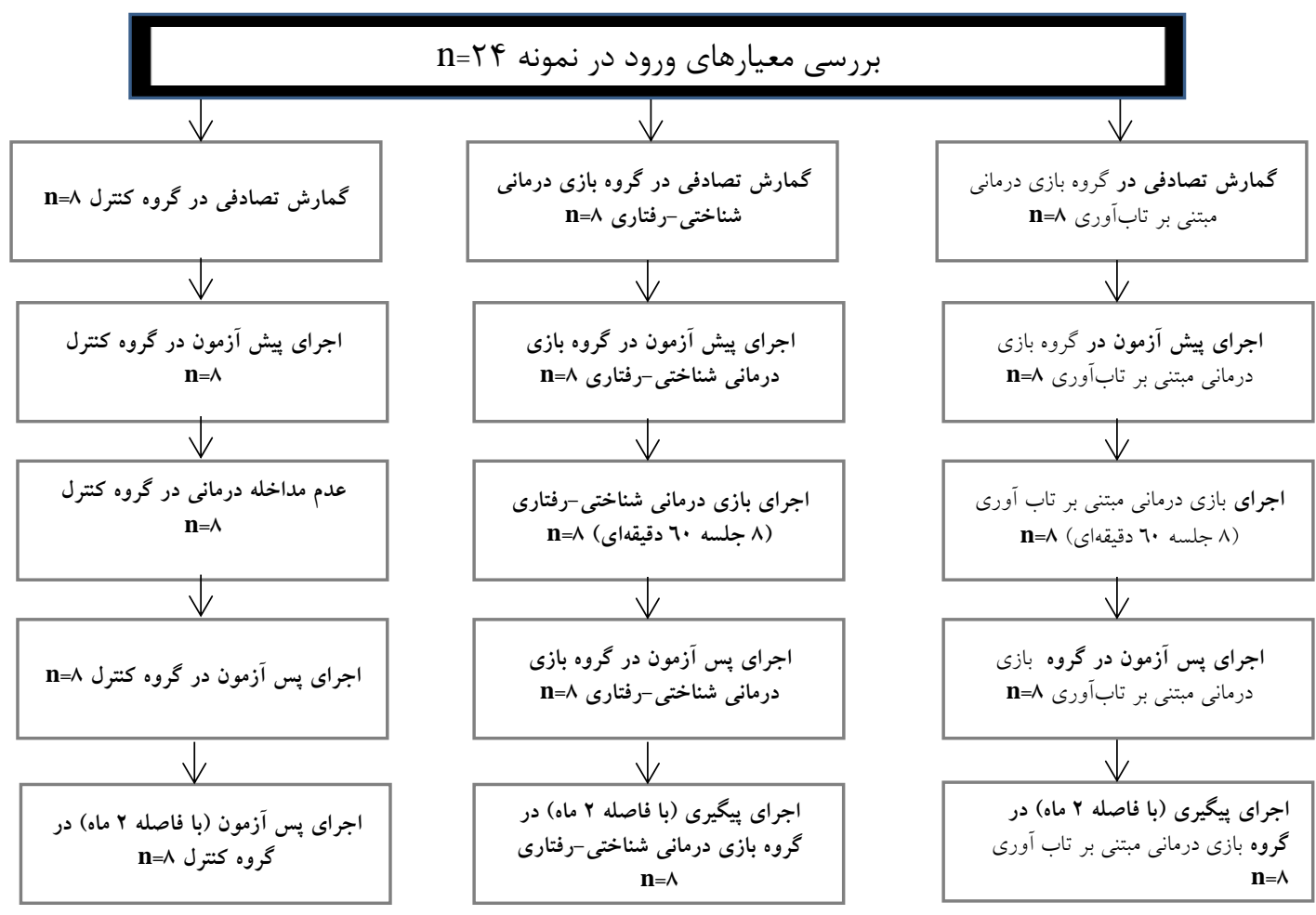

نمودار ا. نمودار CONSORT

جدول ا. خلاصه جلسات بازى درمانى مبتنى بر تاب آورى. (RPT) هدف

ايجاد رابطه اعتمادآميزدرمانكر با كودى و اعضاء با يكديخر، قصه كويى در مورد درد شكمى كودكان توسط دو عروسك و كارهايى كه در آن موقعيت انجام مى دهند.

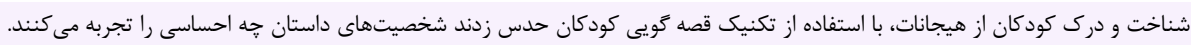

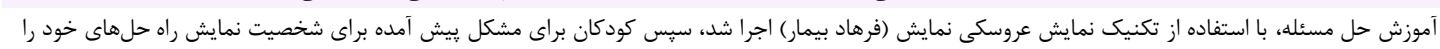
بيان نمودند. آموزش رفتار جرأتمندانه و افزايش عزت نفس، با ايفاى نقش به تمرين رفتار جرأتمندانه يرداختند و با استفاده از عروسك انگشتى و اجراى نمايش تفاوت رفتار يرخاشكرانه،

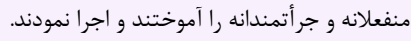

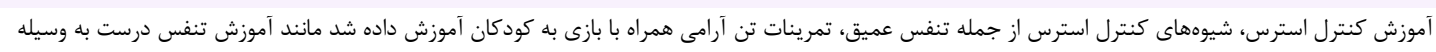

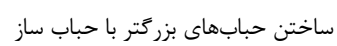

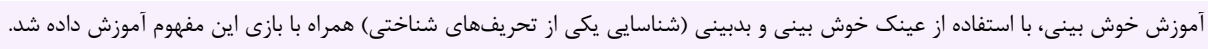

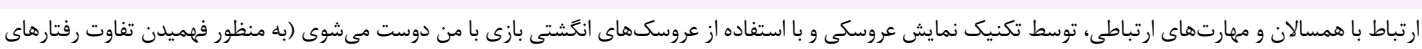

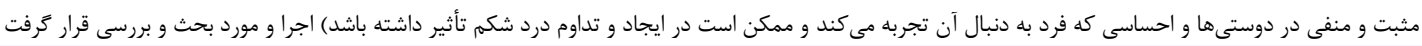

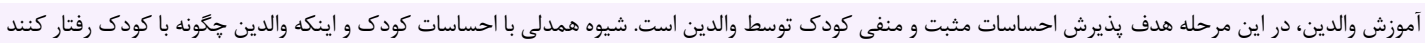

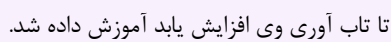


جدول r. خلاصه جلسات بازى درمانى شناختى -رفتارى (CBPT)

\begin{tabular}{|c|c|}
\hline هدف & جلسات \\
\hline 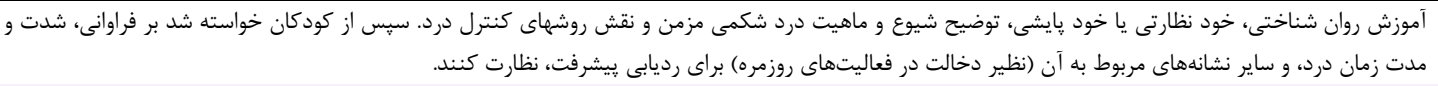 & اول \\
\hline شانتوميم احساساتى هيجاتات، با استفاده از تصاوير كارتونى كه در آن هيجان خاصى نشان داده مىشد كودكان حدس زدند فرد مورد نظر خه احساسى را تجربه مى كند و سِس با اجراى & دوم \\
\hline 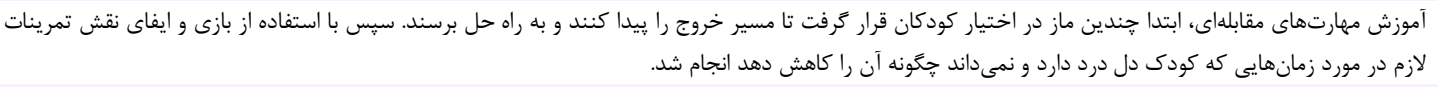 & سوم \\
\hline تمرين تنفس عميق، به كودكان تنفس ديافراگمى از طريق بازى آموزش داده شد (به عنوان مثال، تصوركنند كه يك بادكنك را در معدهان ير و خالى مى كنند) & جهارم \\
\hline آرامش عضلانى يِشرفته، به عنوان مثال، از كودى خواسته شد وانمود كند يك "ربات" و سيس "عروسك پِارجهاى" است. & ينجم \\
\hline 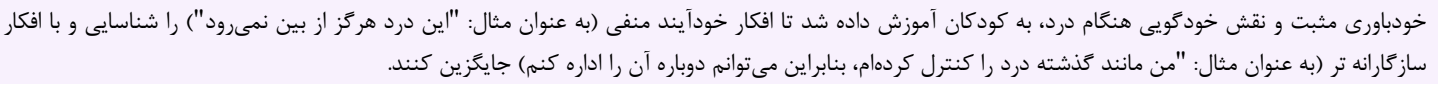 & ششم \\
\hline 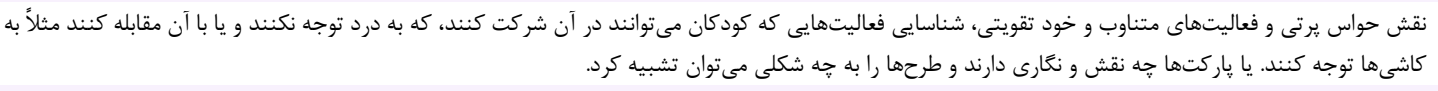 & هفتم \\
\hline 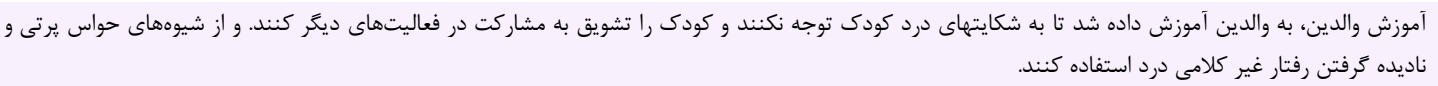 & هشتم \\
\hline
\end{tabular}

نشان مىدهد. بِيش از اجراى تحليل واريانس اندازههاى تكرار شده

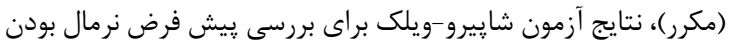

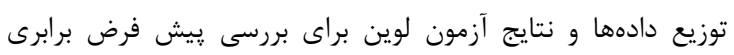

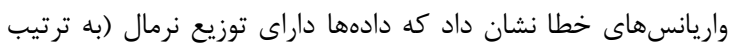

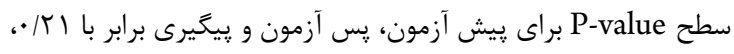

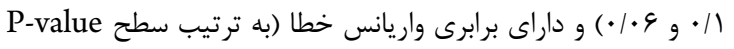

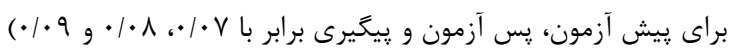

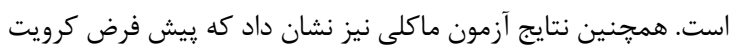

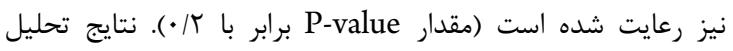

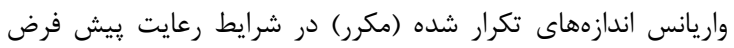
كرويت براى اختلال خواب در جدول ه ارائه شده است.
نتايج مربوط به سن، تعداد خواهران و برادران، تحصيلات يدر و

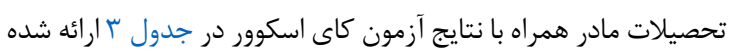

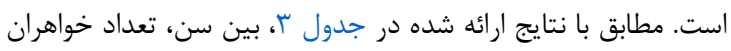

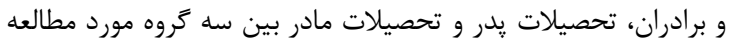

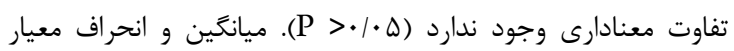

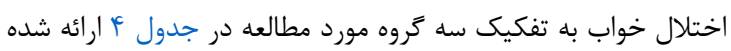

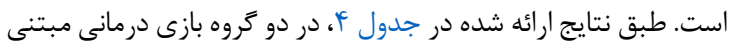
بر تاب آورى (RPT) و كروه بازى درمانى شناختى -رفتارى (CBPT)

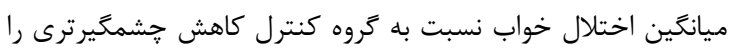

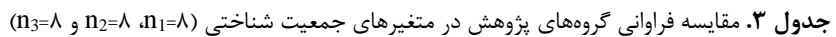

\begin{tabular}{|c|c|c|c|c|c|}
\hline P-Value & مقدار كاى اسكوور & كروه كنترل & كروه CBPT & Rروه RPT & متغير \\
\hline \multirow[t]{3}{*}{.$/ 99$} & r & فراوانى (\% فراوانى) & فراوانى (٪ فراوانى) & فراوانى (٪ فراوانى) & سن \\
\hline & & $(/ . \Delta \cdot)^{f}$ & $(/ / \Gamma V / \Delta) r$ & $(/ / r V / \Delta) r$ & تال V Vال \\
\hline & & $(/ . \Delta \cdot)^{f}$ & $(1.9 r / \Delta) \Delta$ & $(1.9 \% / \Delta) \Delta$ & ^ تا • ا سال \\
\hline \multirow[t]{3}{*}{$(\cdot / 9) \cdot 195$} & $(1 / \cdot 9) T / T F Q$ & & & & تعداد خواهران و برادران" \\
\hline & & $(/ V \Delta, \% / r V / \Delta) \&, r$ & $(/ \Delta, \% \cdot \Delta \cdot)^{f},{ }^{\varphi}$ & $(/ . \Delta \cdot, \% V \Delta) q^{q} q$ & هيج خواهر و برادر \\
\hline & & $(/ r \Delta, \% / g r / \Delta) r, \Delta$ & $(/ . \Delta, \%(\Delta) r, r$ & $(/ . \Delta \cdot, \% r \Delta) F, r$ & ا خواهر يا برادر \\
\hline \multirow[t]{3}{*}{$\cdot / 19$} & $1 / \pi 4$ & & & & تحصيلات يدر \\
\hline & & $(/ / \mathrm{NV} / \Delta) \mathrm{V}$ & $(/ .9 T / \Delta) \Delta$ & $(/ V \Delta) \varphi$ & تادييلم \\
\hline & & $(\mid r / \Delta) \mid$ & $(/ / r V / \Delta) r$ & $(/ . T \Delta) r$ & بالاتر از دييلم \\
\hline \multirow[t]{3}{*}{.199} & T/QT & & & & تحصيلات مادر \\
\hline & & $(/ / N V / \Delta) V$ & $(/ .9 Y / Q) \Delta$ & $(/ . A V / \Delta) V$ & تاديبلم \\
\hline & & $(/ . \mid r / \Delta) 1$ & $(r V / \Delta) r$ & $(/ / \Gamma / \Delta) 1$ & بالاتر از دييلم \\
\hline
\end{tabular}

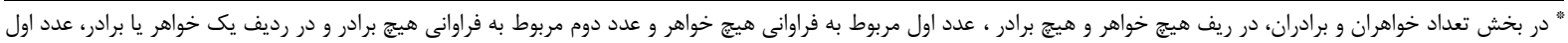

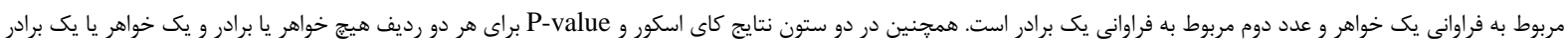
مقادير داخل يرانتز مربوط به هيج برادر و و يا يك برادر درو بـ فراست.

\begin{tabular}{|c|c|c|c|}
\hline & \multicolumn{3}{|c|}{ جدول F. ميانكَين و انحراف استاندارد متغير اختلال خواب در سه كروه يُروهش در سه مرحله زمانى } \\
\hline CBPT & RPTor & تروه كنترل & مرحله \\
\hline$r \Delta / v \Delta \pm 11 / \pi q$ & $F \Delta / V \Delta \pm \Delta / V$ & $\Psi N / V D \pm|\Psi /| \varphi$ & ي ي آزمون \\
\hline$r \Delta \pm r / 99$ & $r F / / r \pm r / r \varepsilon$ & $\Delta f \pm I V$ & 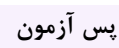 \\
\hline$r F / \Lambda V \pm r / r q$ & $r r^{\prime} \pm r / \cdot V$ & $\Delta / / A V \pm 11 / 94$ & 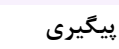 \\
\hline
\end{tabular}




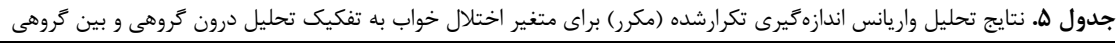

\begin{tabular}{|c|c|c|c|c|c|c|c|}
\hline توان آزمون & مجذور سهمى اتا & معنادارى & ضريبF & ميانكين مجذورات & درجه آزادى & مجموع مجذورات & تحليل \\
\hline & & & & & & & درون تروهى \\
\hline 1 &.$/ 48$ &.$/ . .1$ & $1 V / 99$ & TrN/AD & r & \&VV/9q & زمان (سه مرحله) \\
\hline 1 & . &.$/ .1$ & W/AF & THEIVE & r & $9 \cdot v / \cdot 9$ & تعامل كروه و زمان \\
\hline \multirow[t]{2}{*}{-} & - & - & - & $19 / 19$ & kT & $\Lambda \cdot r / \Delta \Lambda$ & خطا \\
\hline & & & & & & & بين كروهى \\
\hline - IAF & $\cdot / r v$ & $\cdot / \cdot 1$ & $9 / 14$ & $1 F \vee q / .9$ & r & rqهA/II & كروه \\
\hline- & - & - & - & $r F \cdot / v q$ & ri & $\Delta \cdot \Delta s / \Delta F$ & خطا \\
\hline
\end{tabular}

جدول \$. نتايج آزمون بونفرونى براى مقايسه دو به دو كروههاى برُوهش در متغير اختلال خواب

\begin{tabular}{|c|c|c|c|c|}
\hline معنادارى & خطاى استاندارد & تفاوت ميانكينها & كروه مورد مقايسه & كروه مبنا \\
\hline.$/ \cdot 14$ & $F / 4 \Lambda$ & 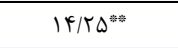 & RPT كروه & كروه كنترل \\
\hline$\cdot / r \Lambda$ & $\uparrow / \Psi_{A}$ & $\mid r / A r^{* *}$ & $\mathrm{CBPT}_{\circ g}$ & تروه كنترل \\
\hline 1 & $\varphi / 4 \wedge$ & $-1 / 4 T$ & $\mathrm{CBPT}_{0}$ & RPT تروه RT \\
\hline
\end{tabular}

تأييد شده است. در تطبيق يافتهاى يزوهش حاضر با يافتههاى

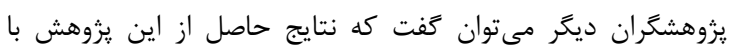

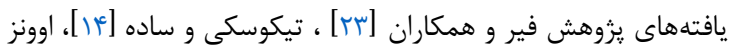

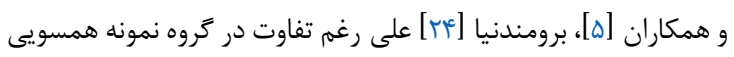

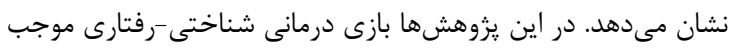

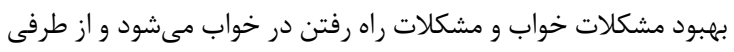

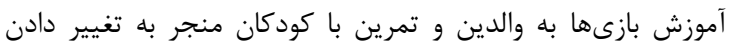

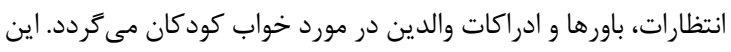

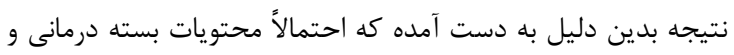

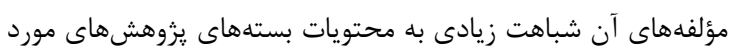

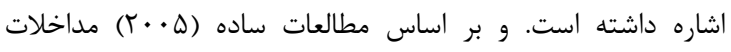

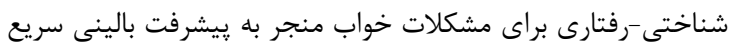

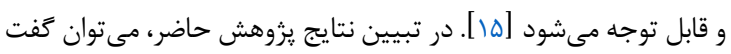

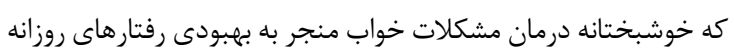

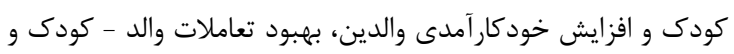

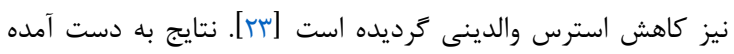

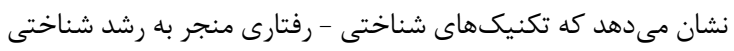

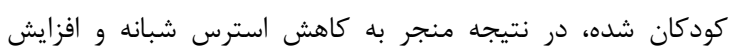

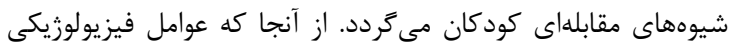

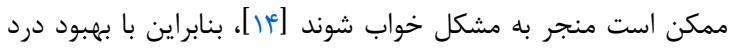
شكمى كودكان كروه نمونه اختلالات خواب كودكان نيز كاهش ئ يافته

مطالعات نشان داده كه بين مشكلات خواب و تاب آورى در كود كان و

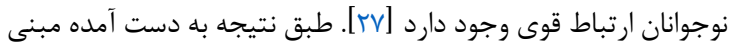

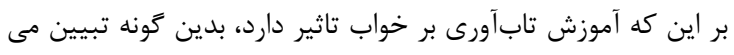

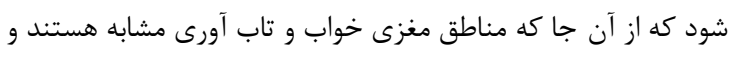

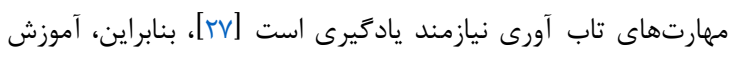

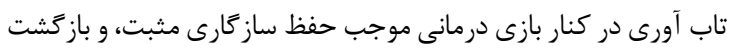

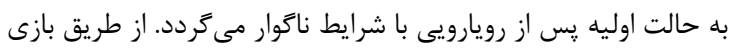

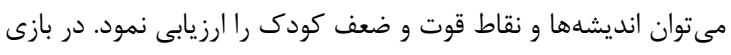

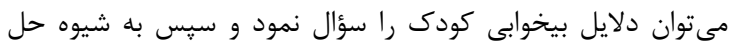

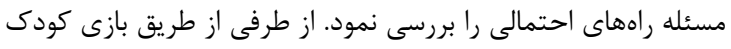

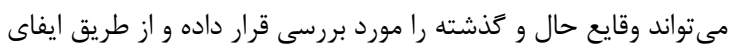

طبق يافته هاى ارائه شده در جدول ه، در بخش تحليل بين گروهى،

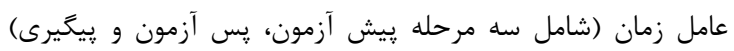

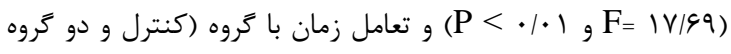
آزمايش) (P ) F

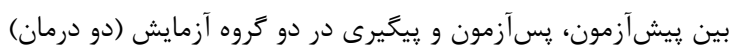

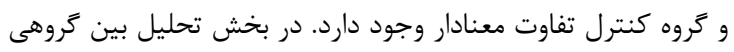

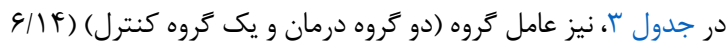

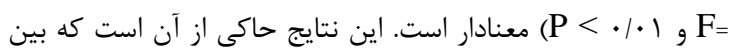

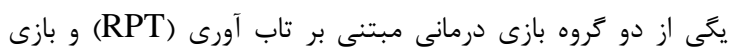

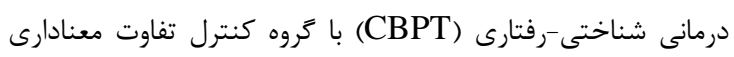

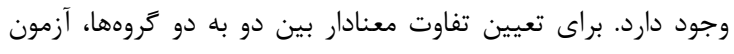

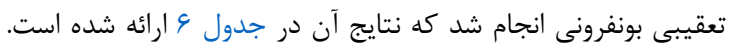

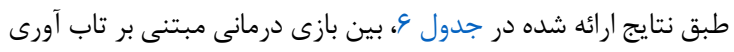
و بازى درمانى شناختى رفتارى (RPT)

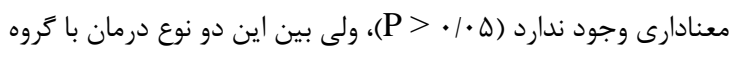

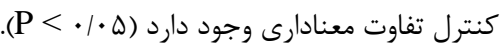

هدف يزوهش بررسى اثر بخشى بازى درمانى مبتنى بر تاب آورى و

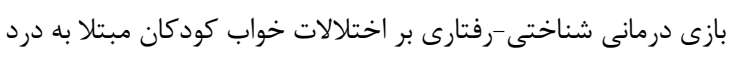

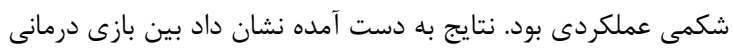

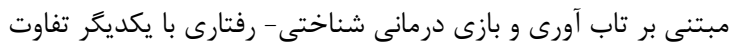

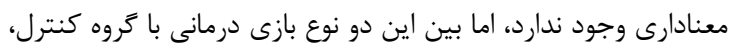

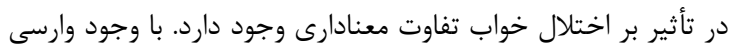

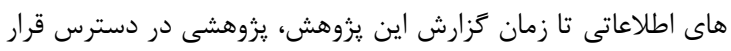

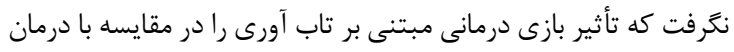

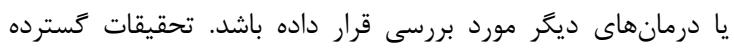

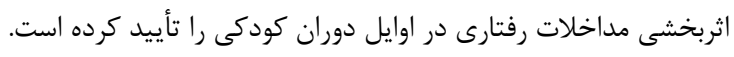

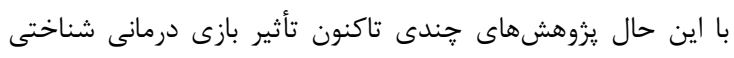

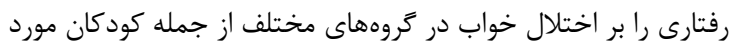
بر برسى قرار دادهاند. اثربخشى بازى درمانى شناختى -رفتارى در بهبود دادان اختلال خواب در

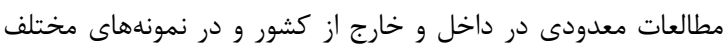


IR.IAU.KHUISF. REC. يروتكلهاى اخلاقى مطالعات هلسينكى عمل نمودند. اطلاعات بيماران

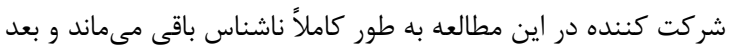

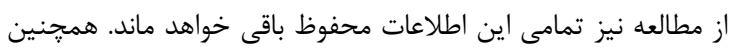

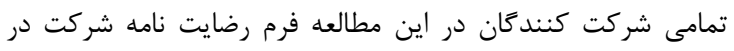

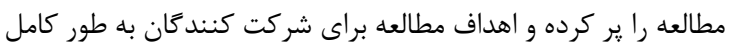
شرح داده شده است.

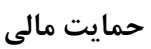
يروهش انجام شده بدون دريافت هيج كَونه كمك مالى از مؤسسه يا سازمانى خاص انجام شده است. سهمم نويسندكًان خانم شكوفه نيك نشان نعارش اوليه و تهيه درافت مقاله را بر عهده

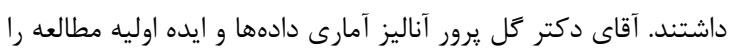

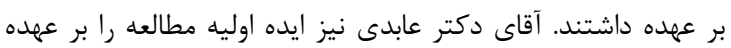

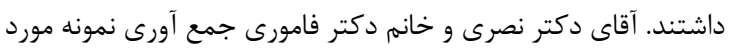
نظر را به عهده كرفتند.

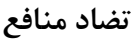
هيج كَنه تضاد منافعى بين نويسند كاربرد عملى مطالعه اين مطالعه با بررسى نقش بازى درمانى شناختى ماركيه -رفتارى و بازى درمانى

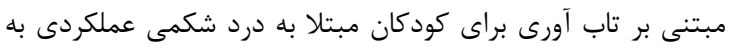
بهبود خواب آنان كمك خواهد كرد. سياسگزارى

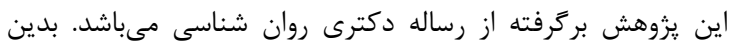

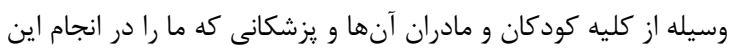
يروهش خالصانه يارى نمودند، صميمانه تشكر و قدردانى مى نمائيم
نقش، داستان گَويى، و يا تصويرسازى ذهنى به حل مسائل خود بيردازد.

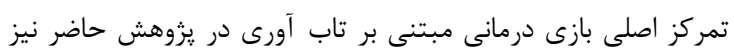

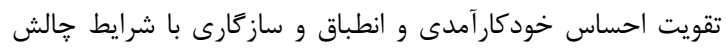

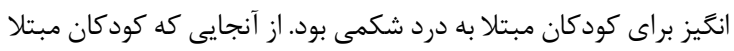

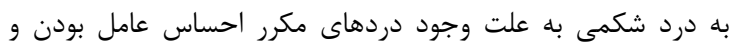

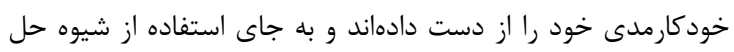

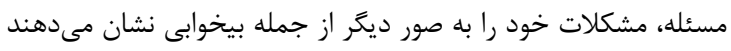

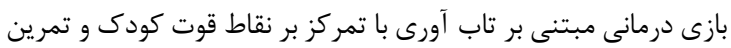

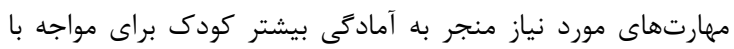

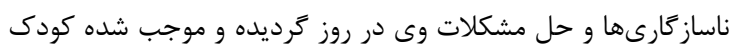

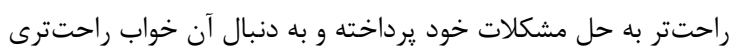
داشته باشد.

محدود بودن جامعه يزوهش به كودكان ه تا • ا سال و كودكان مبتلا

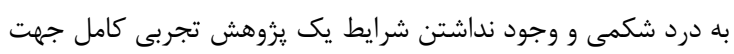

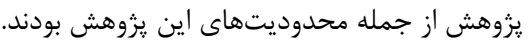

\section{نتيجه كيرى}

يافتههاى يزوهش حاضر نشان داد كه بازى درمانى شناختى رفتارى و

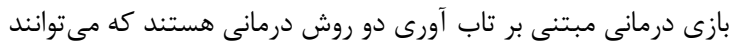

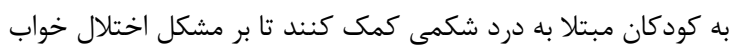

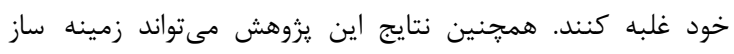

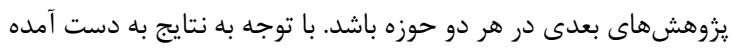

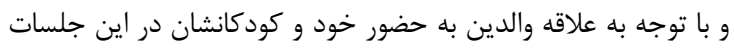

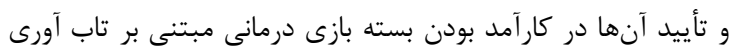

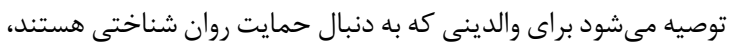

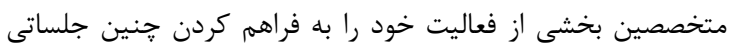
اختصاص دهند و مشوق والد و كودى در بيخيَيرى آن باشند.

ملاحظات اخلاقى

اين يزوهش بركرفته از رساله دكترى روان شناسى و داراى كد اخلاق

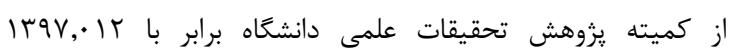

J Dev Behav Pediatr. 2000;21(1):27-36. doi: 10.1097/00004703-200002000-00005 pmid: 10706346

6. Lynch MK, Dimmitt RA, Goodin BR. Evidence of Disturbed Sleep in Children With Eosinophilic Esophagitis and Persistent Epigastric Pain. J Pediatr Psychol. 2018;43(3):331-41. doi: 10.1093/jpepsy/jsx117 pmid: 29048495

7. Beebe DW. Cognitive, behavioral, and functional consequences of inadequate sleep in children and adolescents. Pediatr Clin North Am. 2011;58(3):649-65. doi: $10.1016 /$ j.pcl.2011.03.002 pmid: 21600347

8. Smaldone A, Honig JC, Byrne MW. Sleepless in America: inadequate sleep and relationships to health and wellbeing of our nation's children. Pediatrics. 2007;119 Suppl 1:S29-37. doi: 10.1542/peds.2006-2089F pmid: 17272582 
9. Khanijow V, Prakash P, Emsellem HA, Borum ML, Doman DB. Sleep dysfunction and gastrointestinal diseases. Gastroenterol Hepatol. 2015;11(12):817.

10. Hyun MK, Baek Y, Lee S. Association between digestive symptoms and sleep disturbance: a cross-sectional community-based study. BMC Gastroenterol. 2019;19(1):34. doi: 10.1186/s12876-019-0945-9 pmid: 30782128

11. Valrie CR, Bromberg MH, Palermo T, Schanberg LE. A systematic review of sleep in pediatric pain populations. J Dev Behav Pediatr. 2013;34(2):120-8. doi: 10.1097/DBP.0b013e31827d5848 pmid: 23369958

12. Allen JM, Graef DM, Ehrentraut JH, Tynes BL, Crabtree VM. Sleep and Pain in Pediatric Illness: A Conceptual Review. CNS Neurosci Ther. 2016;22(11):880-93. doi: 10.1111/cns. 12583 pmid: 27421251

13. Pavlova M, Ference J, Hancock M, Noel M. Disentangling the Sleep-Pain Relationship in Pediatric Chronic Pain: The Mediating Role of Internalizing Mental Health Symptoms. Pain Res Manag. 2017;2017:1586921. doi: 10.1155/2017/1586921 pmid: 29348713

14. Tikotzky L, Sadeh A. The role of cognitive-behavioral therapy in behavioral childhood insomnia. Sleep Med. 2010;11(7):686-91. doi: 10.1016/j.sleep.2009.11.017 pmid: 20620108

15. Sadeh A. Cognitive-behavioral treatment for childhood sleep disorders. Clin Psychol Rev. 2005;25(5):612-28. doi: 10.1016/j.cpr.2005.04.006 pmid: 15979219

16. Ritzi RM, Ray DC, Schumann BR. Intensive short-term child-centered play therapy and externalizing behaviors in children. Int J Play Therap. 2017;26(1):33. doi: 10.1037/pla0000035

17. Jones EM, Landreth G. The efficacy of intensive individual play therapy for chronically ill children. Int J Play Therap. 2002;11(1):117. doi: 10.1037/h0088860

18. Faure C, Thapar N, Di Lorenzo C. Pediatric neurogastroenterology: gastrointestinal motility and functional disorders in children.: Translated: Springer; 2016.

19. Bratton SC, Ray D, Rhine T, Jones L. The efficacy of play therapy with children: A meta-analytic review of treatment outcomes. Profession Psychol Res Pract. 2005;36(4):376. doi: 10.1037/0735-7028.36.4.376

20. Pender RR, Webber J, Mascari JB. Using Play to Help Children Cope With Chronic Pain: A New Role for Counselors.

21. Knell SM. Cognitive-behavioral play therapy. Handbook Play Therap. 2015:119. doi: 10.1002/9781119140467.ch6

22. Senko K, Bethany H. Play Therapy: An Illustrative Case. Innovation Clinic Neurosci. 2019;16(5-6):38.
23. Fehr KK, Russ SW, Ievers-Landis CE. Treatment of sleep problems in young children: A case series report of a cognitive-behavioral play intervention. Clinic Pract Pediatr Psychol. 2016;4(3):306. doi: 10.1037/cpp0000153

24. Broumandnia P. Comparison of the Effectiveness of Behavioral Education and Combining Behavioral Education and Cognitive Parental Reconstruction in Reducing Symptoms of Sleep Problems in Children and Improving Maternal Sleep Quality: Ferdowsi University of Mashhad; 2016.

25. El Rafihi-Ferreira R, Pires MLN, de Mattos Silvares EF. Behavioral intervention for sleep problems in childhood: a Brazilian randomized controlled trial. Psicol Reflex Crit. 2019;32(1):5. doi: 10.1186/s41155-019-0118-3 pmid: 32026011

26. Doi S, Fujiwara T, Ochi M, Isumi A, Kato T. Association of sleep habits with behavior problems and resilience of 6to 7-year-old children: results from the A-CHILD study. Sleep Med. 2018;45:62-8. doi: 10.1016/j.sleep.2017.12.015 pmid: 29680430

27. Chatburn A, Coussens S, Kohler MJ. Resiliency as a mediator of the impact of sleep on child and adolescent behavior. Nat Sci Sleep. 2013;6:1-9. doi: 10.2147/NSS.S54913 pmid: 24379734

28. Lester P, Saltzman WR, Woodward K, Glover D, Leskin GA, Bursch B, et al. Evaluation of a family-centered prevention intervention for military children and families facing wartime deployments. Am J Public Health. 2012;102 Suppl 1:S48-54. doi: 10.2105/AJPH.2010.300088 pmid: 22033756

29. Gillham JE, Reivich KJ, Freres DR, Chaplin TM, Shatte AJ, Samuels B, et al. School-based prevention of depressive symptoms: A randomized controlled study of the effectiveness and specificity of the Penn Resiliency Program. J Consult Clin Psychol. 2007;75(1):9-19. doi: 10.1037/0022-006X.75.1.9 pmid: 17295559

30. Bruni O, Ottaviano S, Guidetti V, Romoli M, Innocenzi M, Cortesi F, et al. The Sleep Disturbance Scale for Children (SDSC). Construction and validation of an instrument to evaluate sleep disturbances in childhood and adolescence. J Sleep Res. 1996;5(4):251-61. doi: 10.1111/j.1365-2869.1996.00251.x pmid: 9065877

31. Chambers CT, Holly C, Eakins D. Cognitive-behavioural treatment of recurrent abdominal pain in children: A primer for paediatricians. Paediatr Child Health. 2004;9(10):705-8. doi: 10.1093/pch/9.10.705 pmid: 19688079

32. Nikneshan S, Abedi A, Golparvar M, Famouri F, Nasri P. Developing a model for resilience training special for children with functional abdominal pain syndrome: a qualitative approach. Govaresh. 2019;24(1):30-9. 